Revista Destaques Acadêmicos, Lajeado, v. 10, n. 3, 2018. ISSN 2176-3070

DOI: http://dx.doi.org/10.22410/issn.2176-3070.v10i3a2018.1955

http://www.univates.br/revistas

\title{
SAÚDE DO TRABALHADOR EM UMA ESTRATÉGIA DE SAÚDE DA FAMÍLIA
}

\author{
Andréia Ghiggi ${ }^{1}$, Paula Michele Lohmann ${ }^{2}$, Luís Felipe Pissaia ${ }^{3}$, \\ Arlete Eli Kunz da Costa ${ }^{4}$
}

\begin{abstract}
Resumo: O objetivo deste estudo foi avaliar a saúde de profissionais de uma Estratégia Saúde da Família de um município do interior do Rio Grande do Sul, Brasil. Trata-se de um estudo de abordagem qualitativa, descritiva e exploratória, na qual participaram oito profissionais. Os dados foram coletados por meio de entrevistas e posteriormente foram analisados conforme a Análise do Conteúdo de Bardin. A maioria dos informantes consideram sua saúde como sendo ótima, boa, não muito boa ou afetada e que existem fatores de estresse na unidade em que atuam. Quando questionados sobre o estresse associado ao ambiente de trabalho, os participantes consideram que estão sob nível de estresse que varia de regular a alto. A maioria dos participantes referiu sentir-se ansioso e cansado com as atividades do trabalho e em sua maioria, não apresentam patologias que considerem estar relacionadas ao seu trabalho, porém as principais doenças relatadas foram a ansiedade e a depressão.
\end{abstract}

Palavras-chave: Estratégia Saúde da Família; Saúde do trabalhador; Equipe de Saúde.

\section{INTRODUÇÃO}

A Atenção Primária a Saúde (APS) se caracteriza como sendo um conjunto de ações em saúde, individual e coletiva, que engloba a proteção e a promoção da saúde. Além da prevenção de danos, tratamento, diagnóstico, reabilitação, manutenção da saúde e a redução de agravos, que tem como objetivo otimizar uma atenção integral de saúde e autonomia das pessoas (BRASIL, 2012). Segundo o Ministério da Saúde (MS), a Estratégia Saúde da

1 Acadêmica de Enfermagem. Universidade do Vale do Taquari - UNIVATES.

2 Enfermeira. Doutoranda em Ambiente e Desenvolvimento. Universidade do Vale do Taquari - UNIVATES.

3 Enfermeiro. Mestrando em Ensino. Universidade do Vale do Taquari - UNIVATES.

4 Enfermeira. Doutora em Ambiente e Desenvolvimento. Universidade do Vale do Taquari UNIVATES. 
Família (ESF) tem o papel de prestar uma assistência contínua à comunidade. Fazendo o acompanhamento à saúde da criança, do adulto, da mulher, do idoso, e de todas as pessoas do território que vivem ante sua responsabilidade (BRASIL, 2001).

Segundo Azambuja et al (2007), o contexto do trabalho para os profissionais de saúde é permeado por constantes desafios diários que acabam afetando suas vidas. Silva e Barros (2015) afirmam que compreender a realidade das pessoas que trabalham na APS faz-se necessário para valorizar as equipes de saúde em sua prática profissional, contribuindo na promoção de saúde e prevenção de doenças, uma vez que os funcionários lidam com a população.

De acordo com Lima et al (2014), o relacionamento entre o profissional e usuário, se for verdadeiro, terá impacto positivo não só para o usuário e a comunidade, mas para o trabalhador também. Segundo Silva (2011), o homem atualmente não busca apenas a saúde, mas sim a qualidade de vida, e como profissional, não deseja boas condições apenas para desempenhar suas atividades, mas sim, qualidade de vida no trabalho.

Presoto (2008) afirma que a proposta de um trabalho humanizado será possível somente a partir do reconhecimento do trabalhador, no processo do trabalho. O produto não poderá ter qualidade, sem antes reconhecer a qualidade do produtor e só será possível oferecer serviço humanizado ao usuário, após garantir uma atenção humanizada aos trabalhadores. Neste contexto, o estudo possui o objetivo de avaliar a saúde dos profissionais de uma Estratégia Saúde da Família de um município do interior do Rio Grande do Sul, Brasil.

\section{METODOLOGIA}

Trata-se de uma pesquisa qualitativa, descritiva e exploratória. Os participantes da pesquisa foram oito profissionais da equipe de saúde, atuantes emu ma ESF de um município do interior do Rio Grande do Sul, Brasil.

Conforme a observação em campo, a ESF oferece atendimento clínico, nutricional, ginecológico, odontológico e de enfermagem. Conta com profissionais especialistas que têm parceria com a Secretária de Saúde para especialidades: cardiologista, cardiovascular, dermatologista, endócrino, gastroenterologista, neurologista, oftalmologista, ortopedista, traumatologista, otorrino, pneumologista, psiquiatra, reumatologista e urologista. Realiza-se o agendamento de consultas, a demanda espontânea, a triagem e atendimento de urgência.

A coleta de dados foi realizada no período de fevereiro a abril de 2018, após aprovação da Secretaria Municipal de Saúde do município, sendo então visitada a unidade e agendada a entrevista com os profissionais, momento em que eles responderam à questões semiestruturadas que continha seis perguntas, sendo elas: Como você avalia sua saúde?; Como está seu nível de estresse no ambiente de trabalho?; Quanto à ansiedade, você se considera 
uma pessoa ansiosa?; Você considera que está esgotado com as tarefas do seu trabalho no dia a dia?; Faz uso de algum medicamento?; Se sim, faz uso para qual patologia?.

As entrevistas foram individuais e tiveram duração máxima de trinta minutos, sendo que seus áudios foram gravados, posteriormente transcritos e analisados conforme propõe a Análise de Conteúdo de Bardin (2011).

As questões éticas foram preservados por meio da exclusão dos nomes dos participantes e a inserção de codinomes identificados pela siga P.E., seguida por números ordinais sorteados aleatoriamente. Em todas as etapas da pesquisa foram respeitados os preceitos éticos contidos na Resolução 466/12.

\title{
RESULTADOS E DISCUSSÃO
}

A categorização temática gerada na análise dos dados resultou em quatro eixos de discussão: Percepção sobre a minha saúde; Estresse e ambiente de trabalho; Ansiedade e as tarefas do dia a dia e Principais patologias dos trabalhadores.

\section{Percepção sobre a minha saúde}

Na autoavaliação sobre sua saúde, os participantes consideram-na como ótima, boa, não muito boa ou afetada. Como podemos observar em alguns discursos:

\author{
"Minha saúde física está bem, porém a saúde mental encontra-se \\ fragilizada" (P.E. 1) \\ "Afetada" (P.E. 2) \\ “Não muito boa” (P.E. 3)
}

Para os autores Carvalho et al (2017), as tarefas do trabalho causam prejuízos à saúde dos profissionais, à instituição e assistência ao paciente. É necessária ação organizacional, para prevenção de acidentes e doenças associados ao trabalho, reduzindo as cargas e promoção da saúde do profissional de enfermagem. Gomes, Mendes e Fracolli (2016) confirmam a necessidade de uma rede para apoio aos profissionais que atuam na ESF, uma vez que a qualidade de vida deles pode estar prejudicada, podendo influenciar também na qualidade de vida de sua família, colegas de trabalho e usuários do sistema.

Os dois discursos a seguir reproduzidos são também respostas ao questionário feito aos profissionais de saúde sobre sua própria saúde, conforme os relatos abaixo: 
"Boa, pois trabalhando na área da saúde vemos diariamente pessoas com problemas sérios" (P.E. 4)

“Boa" (P.E. 5)

Para os autores Almeida et al (2015), a pessoa ativa para produzir, vai precisar estar em estado físico, mental e psíquico bons, em decorrência desta harmonia, o trabalho realizado será de melhor qualidade e mais produtivo. Segundo Campos, David e Souza (2014), a saúde do trabalhador deve ser estudada de forma interdisciplinar, que permite compreender o trabalho como organização da vida social, em que os profissionais agem e pensam sobre o trabalho, mesmo que tenham dificuldades sobre essas ações.

Paludo et al (2011) afirmam que é necessário a realização de mais estudos que busquem identificar fatores relacionados com a qualidade de vida no ambiente de trabalho, encontrando soluções e medidas fáceis para serem adotadas, proporcionando ao trabalhador alegria, satisfação e desejo na execução de suas tarefas. Guimarães (2013) afirma que os trabalhadores de enfermagem apresentam problemas de saúde causados pela exposição às cargas de trabalho, manifestando-se por inúmeros processos de desgaste, refletindo a condições de trabalho a que estes trabalhadores estão submetidos.

\title{
Estresse e ambiente de trabalho
}

Quando questionados sobre o estresse associado ao ambiente de trabalho, os informantes consideram que estão sob nível de estresse que varia de regular a alto, conforme verificado abaixo:

\author{
"Regular" (P.E. 6) \\ “Consideravelmente alto" (P.E. 7)
}

\begin{abstract}
“Meu trabalho é estressante, pois lidar com pessoas doentes acaba sempre nos deixando cansados mentalmente; posso dizer que o nível de estresse está alto. Não consigo não me preocupar com os problemas dos outros" (P.E. 1)
\end{abstract}

Para os autores Felix et al (2017), nas ações desenvolvidas contra o estresse, espera-se um ambiente adequado à execução do trabalho, e a longo prazo, melhor qualidade de vida do funcionário. Morais et al (2017) afirmam que é necessário o desenvolvimento de medidas para reduzir o efeito negativo do estresse do profissional existente no ambiente de trabalho, minimizando as dificuldades, dando apoio aos trabalhadores, proporcionando melhor condição 
de vida dentro e fora do trabalho, melhorando assim a qualidade da assistência prestada ao usuário. Verificamos tais questões nos relatos abaixo:

“Alto, porque a responsabilidade é grande e as pessoas (pacientes)
estão sempre reclamando, você faz o possível e o impossível, tá
difícil de você agradar, você só escuta problemas e queixas" (P.E. 2)

“Bem elevado" (P.E. 3)

Segundo Souza (2014), através de sinais e sintomas, o estresse se manifesta. A essas manifestações é preciso estar atento e reconhecer quando o profissional está estressado e proporcionar, o quanto antes, o tratamento da doença, visando a recuperação e a volta do profissional às atividades. Monteiro et al (2016) afirmam que a Síndrome de Burnout ou esgotamento profissional é uma resposta à situação de estresse em função de relações entre pessoas, em situações de trabalho afetivas e intensas. Para os autores Cunha et al (2016), no ambiente de trabalho, o estresse está cada vez mais presente, por vivermos de forma acelerada, consequentemente ocasionando uma sobrecarga de compromissos, metas e responsabilidades a serem cumpridas em um curto período de tempo.

\section{Ansiedade e as tarefas do dia a dia}

A maioria dos informantes referiu sentir-se ansioso e cansado com as atividades do trabalho, conforme os relatos abaixo:

“Sim, às vezes" (P.E. 8)

“Com as tarefas não, com as pessoas sim" (P.E. 7)

"Muito; está comigo desde sempre. É um sentimento que chega a ser angustiante" (P.E. 1)

Para Claudino e Cordeiro (2006), a ansiedade pode ser considerada como uma reação natural para a autopreservação. Entretanto, a ansiedade pode ter efeitos negativos para o indivíduo, se for excessiva e de longa duração, pois dificulta, limita ou impossibilita a adaptação. De acordo com os autores Ascari, Schmitz e Silva (2013), o trabalho e a saúde do trabalhador são caracterizados por estágios diferentes de incorporação tecnológica, formas de organização e gestão, entre outras, que refletem nos profissionais em seu dia a dia.

Sob o mesmo limiar, encontram-se os relatos abaixo: 
"Meu trabalho além de assistencial é bastante burocrático, e isso acaba esgotando sim, são deveres diários que precisam ser realizados e nem sempre obtemos retorno" (P.E. 1)

"Sim, porque cada dia você vai encontrar um paciente insatisfeito com algo. Sempre tem algo de novo, as vezes nem estão insatisfeitos com a saúde, com outra coisa, mas é conosco que vão falar" (P.E. 2)

"Sim, como foi comentado você está sempre a espera de entrar um paciente que está estressado, não encontrou algo e você recebe a culpa, você chega em casa esgotado" (P.E. 2)

Segundo o autor Silva (2017) a ansiedade apresenta componentes psicológicos e fisiológicos, sendo um estado emocional que faz parte da experiência humana. E uma reação natural e essencial para a autopreservação, sendo a responsável pela adequação do organismo frente a episódios de perigo. Outros relatos foram verificados, conforme observado abaixo:

"Sim, pois são pessoas queixosas o tempo todo e a maioria vem ao posto precisando de uma palavra de conforto e que necessitam de atenção, e na maioria das vezes temos que fazer o trabalho em excesso" (P.E. 3)

"Sim, porque eu gostaria que as coisas fossem resolvidas com uma certa brevidade, mas nem sempre é como gostaríamos que fosse" (P.E. 4)

"Mais ou menos, porque muitas vezes as pessoas não entendem que não depende de nós e nos sentimos com mãos atadas" (P.E. 4)

“Muito, porém aprendi a controlar" (P.E. 6)

Para os autores Fontinhas e Cardoso (2017), um tema que tem sido estudado é o estresse relacionado ao trabalho, colocando em risco a saúde da pessoa, impactando a vida profissional e pessoal do trabalhador. De acordo com a autora Guimarães (2007), manifesta-se a ansiedade primária como única ou principal de um quadro clínico. E a ansiedade patológica secundária é a decorrência de outras doenças psiquiátricas ou não. A autora afirma ainda que os sintomas de ansiedade são psíquicos e somáticos, variando de acordo com o transtorno. 


\section{Principais patologias dos trabalhadores}

Os profissionais de saúde, em sua maioria, não apresentam patologias que considerem estar relacionadas ao seu trabalho, porém as principais doenças relatadas por eles foram a ansiedade e depressão (4), ansiedade (2) Enxaqueca crônica (1), e o uso de antidepressivos, como por exemplo um informante mencionou o uso de Citalopram e Amitriptilina.

As doenças que afetam os trabalhadores se referem a um conjunto de fatores que são causados por riscos presentes no local de trabalho. A manifestação ocorre de forma lenta, podendo levar até anos para manifestarse, o que dificulta a investigação da doença com o trabalho (BRASIL, 2001). Sobre as patologias que os profissionais podem apresentar, os autores Martins et al (2014) afirmam que o esgotamento profissional ou Síndrome de Burnout se caracteriza pelo estresse emocional, baixa realização profissional, que pode ocorrer com os profissionais de saúde.

Segundo os autores Silva e Barros (2015), o estresse causa transformações no organismo do indivíduo, podendo leva-lo a sérias doenças e inclusive a morte. Os autores relatam ainda, que é fundamental conhecer o que causa o estresse nos profissionais, em especial os que trabalham nas unidades de saúde, que estão expostos a fatores estressores que comprometem o seu bem-estar. De acordo com os autores Santos e Rocha (2012), os trabalhadores são submetidos a vários fatores de risco. As frustrações e experiências dolorosas vividas no trabalho podem desencadear sintomas como a depressão.

Para a autora Jardim (2011), a depressão no século XXI surge como o "mal do século", e esse mal-estar no ambiente de trabalho pode levar ao suicídio. A autora afirma ainda que os sinais da depressão são: a tristeza sem motivo, o desinteresse, o desânimo, a irritabilidade, a insônia e inapetência. A falta de sentido na vida, o sentimento de vazio, se caracterizam como sendo o caso mais grave, chegando a ideias e tentativas suicidas. $\mathrm{O}$ silêncio e a dificuldade de falar são outros aspectos importantes que o deprimido apresenta.

\section{CONSIDERAÇÕES FINAIS}

Os resultados demonstraram que a maioria dos participantes consideram na autoavaliação sobre a sua saúde como sendo ótima, boa, não muito boa ou afetada e que existem fatores de estresse na unidade. Quando questionados sobre o estresse associado ao ambiente de trabalho, os participantes consideram que estão sob nível de estresse que varia de regular a alto. A maioria dos participantes referiu sentir-se ansioso e cansado com as atividades do trabalho e em sua maioria, não apresentam patologias que considerem estar relacionadas ao seu trabalho, porém as principais doenças relatadas foram a ansiedade e depressão.

Observamos através desta pesquisa, a importância de um acompanhamento necessário que se deve ter com os profissionais que atuam 
na área da saúde devido ao seu bem-estar, para que possam desenvolver suas tarefas, da melhor maneira possível, visto que esses profissionais possuem uma rotina estressante e uma carga emocional elevada. Bem como a gestão é importante no acompanhamento das equipes de saúde e do trabalho que estão desenvolvendo em suas respectivas unidades.

\section{REFERÊNCIAS}

ALMEIDA, S. L. D. et al. Promoção a saúde do trabalhador. Caderno de Cultura e Ciência, Ano X, v. 14, n. 1, 2015.

ASCARI, R. A.; SCHMITZ, S. D. S.; SILVA, O. M. D. Prevalência de doenças ocupacionais em profissionais da enfermagem: revisão de literatura. Revista UNINGÁ, v. 15, n. 2, 2013.

AZAMBUJA, E. P. D. et al. Significados do trabalho no processo de viver de trabalhadoras de um programa de saúde da família. Texto Contexto Enferm. Florianópolis, v. 16, n. 1, p.71-79, 2007.

BARDIN, L. Análise de conteúdo. São Paulo: Edições 70, 2011.

BRASIL. Ministério da Saúde. Departamento de Atenção Básica. Guia prático do programa de saúde da família. Brasília; 2001.

BRASIL. Ministério da Saúde. Secretaria de Atenção à Saúde. Departamento de Atenção Básica. Política Nacional de Atenção Básica / Ministério da Saúde. Secretaria de Atenção à Saúde. Departamento de Atenção Básica - Brasília: Ministério da Saúde, 2012.

BRASIL. Ministério da Saúde. Secretaria de Políticas de Saúde. Departamento de Atenção Básica. Departamento de Ações Programáticas e Estratégicas. Área Técnica de Saúde do Trabalhador. Saúde do trabalhador / Ministério da Saúde, Departamento de Atenção Básica, Departamento de Aç̃os Programáticas e Estratégicas, Área Técnica de Saúde do Trabalhador - Brasília: Ministério da Saúde, 2001.

CAMPOS, J. F.; DAVID, H. M. S. L.; SOUZA, N. V. D. D. O. Prazer e sofrimento: avaliação de enfermeiros intensivistas à luz da psicodinâmica do trabalho. Escola Anna Nery Revista de Enfermagem, v. 18, n. 1, 2014.

CARVALHO, D. P. D., et al. Cargas de trabalho e a saúde do trabalhador de enfermagem: revisão integrativa. Cogitare Enferm., v. 22, n. 1, p. 01-11, 2017.

CLAUDINO, J.; CORDEIRO, R. Níveis de Ansiedade e depressão nos alunos do curso de licenciatura em enfermagem: O caso particular dos alunos da Escola Superior de Saúde Portalegre. Educação, Ciência e Tecnologia, 32, 197-210, 2006.

CUNHA, N. C. et al. Estresse dentro das organizações de trabalho. Getec, v. 5, n. 9, p. 1-17, 2016. 
FELIX, D. B. et al. Análise dos níveis de estresse no ambiente hospitalar: Um estudo com professionais da área de enfermagem. Revista de Carreiras e Pessoas, v. 6, n. 2, p. 530-543, 2017.

FONTINHAS, J. E.; CARDOSO, J. M. M. O estresse no trabalho do enfermeiro. Revista UNINGÁ, v. 51, n. 1, 2017.

GOMES, M. F. P.; MENDES, E. D. S.; FRACOLLI, L. A. Qualidade de vida dos professionais que trabalham na estratégia saúde da família. Revista de Atenção a Saúde, v. 14, n. 49, p. 27-33, 2016.

GUIMARÃES, A. L. D. O. Problemas de saúde de trabalhadores de enfermagem.

Dissertação - Pós-Graduação em Gerenciamento em Enfermagem da Escola de Enfermagem da Universidade de São Paulo, 2013.

GUIMARÃES, C. M. Efeito da acupuntura nos sintomas de ansiedade e depressão e nos parâmetros fisiológicos de voluntários adultos. Tese - Universidade Federal de São Paulo - Escola Paulista de Medicina, 2007.

JARDIM, S. Depressão e trabalho: ruptura de laço social. Rev. Bras. Saúde Ocup. São Paulo, v. 36, n. 123, p. 84-92, 2011.

LIMA, C. D. A. et al. Relação profissional-usuário de saúde da família: perspectiva da bioética contratualista. Rev. Bioét. (Impr), v. 22, n. 1, p. 152-60, 2014.

MARTINS, L. F. et al. Esgotamento entre profissionais da Atenção Primária a Saúde. Ciência \& Saúde Coletiva, v. 19, n. 12, p. 4739-4750, 2014.

MONTEIRO, J. K. et al. Fatores associados a Síndrome de Burnout em profissionais que tratam da saúde da mulher. Revista de Psicologia da IMED, v. 8, n. 1, p. 3-13, 2016.

MORAIS, A. F. D. et al. Nível de estresse e satisfação dos profissionais da equipe de saúde da família. Anais da INESC - Mostra Científica do Curso de Medicina, v. 1, n. $1,2017$.

PALUDO, C. D. S. et al. Análise da qualidade de vida do ambiente de trabalho dos funcionários da faculdade Anhanguera de Rio Grande. Ensaios e Ciência: Ciências Biológicas, Agrárias e da Saúde, v. 15, n. 4, p. 105-115, 2011.

PRESOTO, L. H. Promoção da saúde e qualidade de vida do trabalhador em hospitais estaduais da cidade de São Paulo. Tese de doutorado. São Paulo: Faculdade de Saúde Pública da USP; 2008.

SANTOS, F. P.; ROCHA, M. A. H. D. Depressão ocupacional: impacto na saúde mental do colaborador. Brazilian Journal of Health, v. 3, n. 2, 2012.

SILVA, D. V. D. Ansiedade, estresse, depressão e uso de drogas entre trabalhadores de enfermagem no ambiente hospitalar. Dissertação - Programa de Pós-Graduação 
Mestrado Profissional em Saúde Ambiental e Saúde do Trabalhador da Universidade Federal de Uberlândia, Instituto de Geografia (PPGAT), 2017.

SILVA, G. O. C.D. O meio ambiente do trabalho e o princípio da dignidade da pessoa humana. eGov - Portal de e-governo, inclusão digital e sociedade do conhecimento. 2011.

SILVA, M. G. D.; BARROS, B. P. D. Percepção de estresse de servidores na Atenção básica de saúde de dourados-ms. Saúde em Redes, v. 1, n. 4, p. 35-52, 2015.

SOUZA, M. M. D. Desgaste e tensão no ambiente de trabalho: Uma proposta de avaliação do nível de estresse da equipe de enfermagem que atua no setor de emergência. Monografia - Curso de Especialização em Linhas de Cuidado em Enfermagem - Urgência e Emergência do Departamento de Enfermagem da Universidade Federal de Santa Catarina, 2014. 